\title{
Madeleine Levasseur
}

Chercheure, Direction de l'évaluation, Ministère de la Santé et des Services sociaux du Québec

\section{(1995)}

\section{"Perception \\ de l'état de santé”}

Un document produit en version numérique par Jean-Marie Tremblay, bénévole, professeur de sociologie au Cégep de Chicoutimi

Courriel : jean-marie_tremblay@uqac.ca

Site web pédagogique : http ://www.uqac.ca/jmt-sociologue/

Dans le cadre de : "Les classiques des sciences sociales" Une bibliothèque numérique fondée et dirigée par Jean-Marie Tremblay, professeur de sociologie au Cégep de Chicoutimi Site web : http ://classiques.uqac.ca/

Une collection développée en collaboration avec la Bibliothèque Paul-Émile-Boulet de l'Université du Québec à Chicoutimi Site web : http ://bibliotheque.uqac.ca/ 
Cette édition électronique a été réalisée par Jean-Marie Tremblay, bénévole, professeur de sociologie au Cégep de Chicoutimi à partir de :

Madeleine Levasseur

“Perception de l’état de santé”.

Un article publié dans l'ouvrage sous la direction de Carmen Bellerose, Claudette Lavallée, Lucie Chénard et Madeleine Levasseur, Santé Québec. Et la santé, ça va en 1992-1993 ? Rapport de l'Enquête sociale et de santé 1992-1993. Volume I. Chapitre 11, pp. 199-209. Québec : Santé Québec, Ministère de la Santé et des Services sociaux, Gouvernement du Québec, novembre 1995, 412 pp.

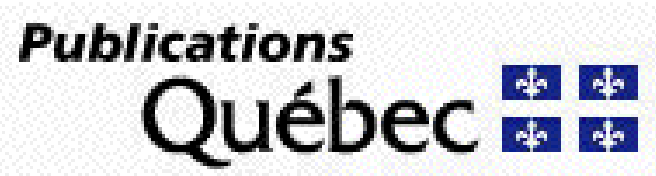

[Au nom de l’Éditeur du Québec, Publications Québec, Mme Cynthia Lachance, responsable de la gestion des droits d'auteur, noua a donné le 17 octobre 2006 la permission de diffuser ce livre, en texte intégral, dans Les Classiques des sciences sociales.]

Courriel : cynthia.lachance@mrci.gouv.qc.ca

Polices de caractères utilisée :

Pour le texte : Times New Roman, 14 points.

Pour les citations : Times New Roman, 12 points.

Pour les notes de bas de page : Times New Roman, 12 points.

Édition électronique réalisée avec le traitement de textes Microsoft Word 2004 pour Macintosh.

Mise en page sur papier format : LETTRE (US letter), 8.5’’ x 11'’)

Édition numérique réalisée le 6 août 2007 à Chicoutimi, Ville de Saguenay, province de Québec, Canada. 
Madeleine Levasseur

Chercheure, Direction de l'évaluation, Ministère de la Santé et des Services sociaux du Québec

\section{“Perception de l'état de santé”.}

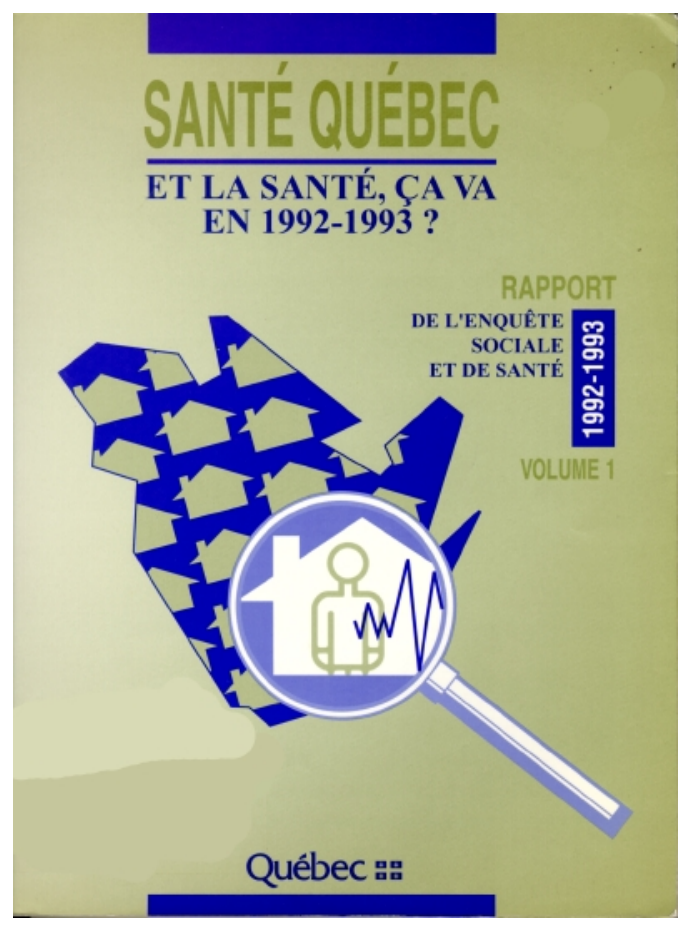

Un article publié dans l'ouvrage sous la direction de Carmen Bellerose, Claudette Lavallée, Lucie Chénard et Madeleine Levasseur, Santé Québec. Et la santé, ça va en 1992-1993 ? Rapport de l'Enquête sociale et de santé 1992-1993. Volume I. Chapitre 11, pp. 199-209. Québec : Santé Québec, Ministère de la Santé et des Services sociaux, Gouvernement du Québec, novembre 1995, 412 pp. 


\section{Table des matières}

11.0 Introduction

11.1 Aspects méthodologiques

11.2 Résultats

11.2.1 Variations selon le sexe et l'âge

11.2.2 Variations selon certaines caractéristiques socioéconomiques

11.2.3 Variations selon certains autres déterminants

11.3 Conclusions

11.3.1 Synthèse et pistes de recherche

11.3.2 Éléments de réflexion pour la planification

11.4 Références bibliographiques 


\author{
Madeleine Levasseur \\ Chercheure, Direction de l'évaluation, \\ Ministère de la Santé et des Services sociaux du Québec \\ "Perception de l'état de santé”.
}

Un article publié dans l'ouvrage sous la direction de Carmen Bellerose, Claudette Lavallée, Lucie Chénard et Madeleine Levasseur, Santé Québec. Et la santé, ça va en 1992-1993 ? Rapport de l'Enquête sociale et de santé 1992-1993. Volume I. Chapitre 11, pp. 199-209. Québec : Santé Québec, Ministère de la Santé et des Services sociaux, Gouvernement du Québec, novembre 1995, 412 pp.

\title{
11. 0 Introduction
}

$\underline{\text { Retour à la table des matières }}$

La perception ou l'autoévaluation de la santé constitue un indicateur important de l'état de santé de la population. Elle reflète l'appréciation globale que l'individu fait de son propre état de santé en intégrant ses connaissances et son expérience de la santé ou de la maladie.

Des études montrent que la perception de l'état de santé est associée non seulement à la morbidité déclarée, sous forme de symptômes ou de maladies aiguës ou chroniques (Fylkesnes et Forde, 1992a ; Liang et al., 1991 ; Segovia et al., 1989b), mais également à la morbidité diagnostiquée (Idler et Angel, 1990 ; Linn et Linn, 1980). La perception de l'état de santé a aussi été reliée aux mesures d'incapacité fonctionnelle et de limitation des activités (Barsky et al., 1992 ; Segovia et al., 1989b). Au surplus, elle est reconnue comme étant un bon prédicteur de la mortalité (Wannamethee et Shaper, 1991 ; Idler et Angel, 1990). Outre les caractéristiques démographiques et so- 
cio-économiques, les comportements individuels, de même que le milieu social, l'utilisation des services de santé et la consommation de médicaments sont aussi associés à la perception de l'état de santé (Santé et Bien-être social Canada, 1993 ; Fylkesnes et Forde, 1992b ; Mays et al., 1992 ; Segovia et al., 1989a ; Kutner, 1987 ; Linn et Linn, 1980).

En l'absence d'une variété d'indicateurs de santé, l'autoévaluation revêt une importance particulière dans la présente enquête. Elle constitue en quelque sorte un indicateur privilégié pour l'analyse de l'état de santé de la population selon ses caractéristiques démographiques et socio-économiques. Dans le prolongement de l'enquête Santé Québec 1987, l'indicateur permet également de suivre l'évolution de l'état de santé général de la population.

\subsection{Aspects méthodologiques}

$\underline{\text { Retour à la table des matières }}$

En plus d'être considérée comme une mesure fiable et valide, comme l'ont souligné Pampalon et al. (1994) dans leur recension des écrits sur le sujet, la perception de l'état de santé comporte l'avantage d'être facile à obtenir. Ne nécessitant qu'une seule question, elle est utilisée dans un grand nombre d'enquêtes de santé dont les enquêtes canadiennes sur la promotion de la santé de 1985 et de 1990 et les enquêtes nationales américaines (NHIS-HPDP, NHANES-1) (Santé et Bien-être social Canada, 1993). Dans la présente enquête, la question fait partie du questionnaire autoadministré (QAAI), et s'adresse à la population de 15 ans et plus. Elle prend la forme suivante : «Comparativement à d'autres personnes de votre âge, diriez-vous que votre santé est en général excellente, très bonne, bonne, moyenne ou mauvaise ? ». Puisque la formulation de la question est la même que celle 
de l'enquête Santé Québec 1987, il est donc possible de comparer les deux enquêtes.

Ce chapitre examine, dans un premier temps, la perception de l'état de santé selon les caractéristiques d'âge et de sexe de la population, et établit des comparaisons avec les données de l'enquête Santé Québec 1987. Dans un second temps, la perception de l'état de santé est examinée en fonction de certaines caractéristiques socio-économiques dont la scolarité relative, le niveau de revenu, le statut d'activité au cours des douze mois ayant précédé l'enquête et la profession ; en fonction également des comportements individuels que sont le tabagisme et l'activité physique, de l'indice de masse corporelle (IMC) et, enfin, selon l'indice de soutien social.

Les données n'étant pas normalisées, sauf dans le cas de la scolarité relative, il faut rappeler l'influence de l'âge sur la plupart des caractéristiques de la population. Le taux de non-réponse partielle est de $0,7 \%$.

\subsection{Résultats}

\subsubsection{Variations selon le sexe et l'âge}

Près de neuf Québécois sur dix (89\%) qualifient leur état de santé de bon à excellent (tableau 11.1). Environ une personne sur cinq (18\%) se dit en excellente santé et un peu plus du tiers de la population se considère en très bonne santé (34\%) ou en bonne santé (37\%). À peine une personne sur dix (9\%) déclare avoir un état de santé moyen, et moins de $2 \%$, une mauvaise santé. 
Chez les hommes comme chez les femmes, la proportion de personnes qui qualifient leur santé d'excellente et de très bonne tend à diminuer avec l'âge, et la situation inverse s'observe chez les personnes qui s'estiment en moyenne et en mauvaise santé. Les personnes de 15-24 ans et de 25-44 ans perçoivent leur état de santé de façon similaire ; les seules différences significatives entre les personnes de ces groupes d'âge s'observent chez les hommes qui, à 25-44 ans, sont moins nombreux à déclarer avoir une excellente santé (21\% c. 28\%) et plus nombreux à se percevoir en bonne santé. C'est principalement à partir de 45 ans que l'évaluation de l'état de santé devient plus négative. Ainsi, la proportion de personnes de 45-64 ans qui jugent leur santé comme étant moyenne ou mauvaise est estimée à $14 \%$, soit environ le double de ce que l'on observe chez les personnes des groupes d'âge plus jeunes ; chez les personnes de 65 ans et plus cette proportion s'élève à $23 \%$.

Dans l'ensemble, les femmes évaluent leur santé un peu plus négativement que les hommes; en particulier, elles sont moins nombreuses (16\% c. $20 \%)$ à qualifier leur état de santé d'excellent. Cette différence selon le sexe est en grande partie attribuable aux personnes de 15-24 ans. Il y a nettement moins de jeunes femmes que de jeunes hommes (18\% c. $28 \%)$ qui s'estiment en excellente santé. Dans les autres groupes d'âge, les proportions présentent peu de variation selon le sexe quel que soit le niveau d'état de santé perçu. 


\section{Tableau 11.1}

Perception de l'état de santé selon le sexe et l'âge, population de 15 ans et plus, Québec 1992-1993

$\underline{\text { Retour à la table des matières }}$

\begin{tabular}{|c|c|c|c|c|c|c|}
\hline \multirow[b]{2}{*}{$\begin{array}{l}\text { Sexe/ } \\
\text { Groupe d'âge }\end{array}$} & \multicolumn{6}{|c|}{ Perception de l'état de santé } \\
\hline & $\begin{array}{c}\text { Excellent } \\
\%\end{array}$ & $\begin{array}{c}\text { Très bon } \\
\%\end{array}$ & $\begin{array}{c}\text { Bon } \\
\%\end{array}$ & $\begin{array}{c}\text { Moyen } \\
\%\end{array}$ & $\begin{array}{c}\text { Mauvais } \\
\%\end{array}$ & $\begin{array}{c}\text { Total } \\
\mathbf{N}\end{array}$ \\
\hline \multicolumn{7}{|l|}{ Hommes } \\
\hline $15-24$ ans & 27,7 & 38,9 & 28,5 & 4,5 & $0,4^{* *}$ & 476611 \\
\hline $25-44$ ans & 21,1 & 37,2 & 35,1 & 6,0 & $0,6^{*}$ & 1235187 \\
\hline $45-64$ ans & 17,5 & 30,8 & 38,0 & 11,1 & 2,5 & 751974 \\
\hline 65 ans et plus & 11,0 & 23,9 & 44,0 & 17,5 & $3,7^{*}$ & 300963 \\
\hline Total & 20,1 & 34,3 & 35,7 & 8,4 & 1,4 & 2764735 \\
\hline \multicolumn{7}{|l|}{ Femmes } \\
\hline $15-24$ ans & 18,0 & 37,6 & 37,6 & 6,4 & $0,4^{* *}$ & 459703 \\
\hline $25-44$ ans & 17,9 & 38,3 & 36,7 & 6,1 & $1,0 *$ & 1222659 \\
\hline $45-64$ ans & 14,0 & 32,6 & 39,1 & 12,5 & $1,9 *$ & 769409 \\
\hline 65 ans et plus & 8,5 & 22,6 & 44,5 & 19,1 & $5,3^{*}$ & 415815 \\
\hline Total & 15,5 & 34,4 & 38,6 & 9,7 & 1,8 & 2867586 \\
\hline \multicolumn{7}{|l|}{ Sexes réunis } \\
\hline $15-24$ ans & 22,9 & 38,3 & 33,0 & 5,5 & $0,4^{* *}$ & 936314 \\
\hline $25-44$ ans & 19,5 & 37,8 & 35,9 & 6,1 & $0,8^{*}$ & 2457846 \\
\hline 45-64 ans & 15,7 & 31,7 & 38,5 & 11,8 & 2,2 & 1521383 \\
\hline 65 ans et plus & 9,5 & 23,2 & 44,3 & 18,4 & 4,6 & 716778 \\
\hline Total & 17,8 & 34,4 & 37,2 & 9,1 & 1,6 & 5632321 \\
\hline
\end{tabular}

* $\quad$ Coefficient de variation entre $15 \%$ et $25 \%$; interpréter avec prudence.

** Coefficient de variation $>25 \%$; estimation imprécise fournie à titre indicatif seulement.

Comparativement à l'enquête Santé Québec 1987, il n'y a pas de différence significative entre la proportion de personnes qui perçoivent leur état de santé comme étant excellent, moyen ou mauvais (graphique 11.1, tableau A. 11.1). Cependant, la proportion de la po- 
pulation qui s'estime en très bonne santé est plus faible qu'en 1987 (34\% c. 41\%) et celle qui s'estime en bonne santé est plus élevée (37\% c. 29\%). Les personnes de 15 à 44 ans sont proportionnellement plus nombreuses en 1992-1993 qu'en 1987 à qualifier leur santé de bonne plutôt que très bonne, tandis que les personnes de 45 ans et plus sont plus nombreuses à qualifier leur santé de bonne plutôt que de la considérer comme étant moyenne ou mauvaise. Chez les personnes de 65 ans et plus, les écarts ne sont pas significatifs entre 1987 et 19921993. L'évolution est semblable pour les hommes et les femmes de 15 à 44 ans, mais, à partir de 45 ans, les hommes évaluent leur santé un peu plus négativement en 1992-1993 qu'en 1987, et les femmes l'évaluent plus favorablement.

\section{Graphique 11.1}

Perception de l'état de santé selon le sexe, population de 15 ans et plus, Québec 1987 et 1992-1993

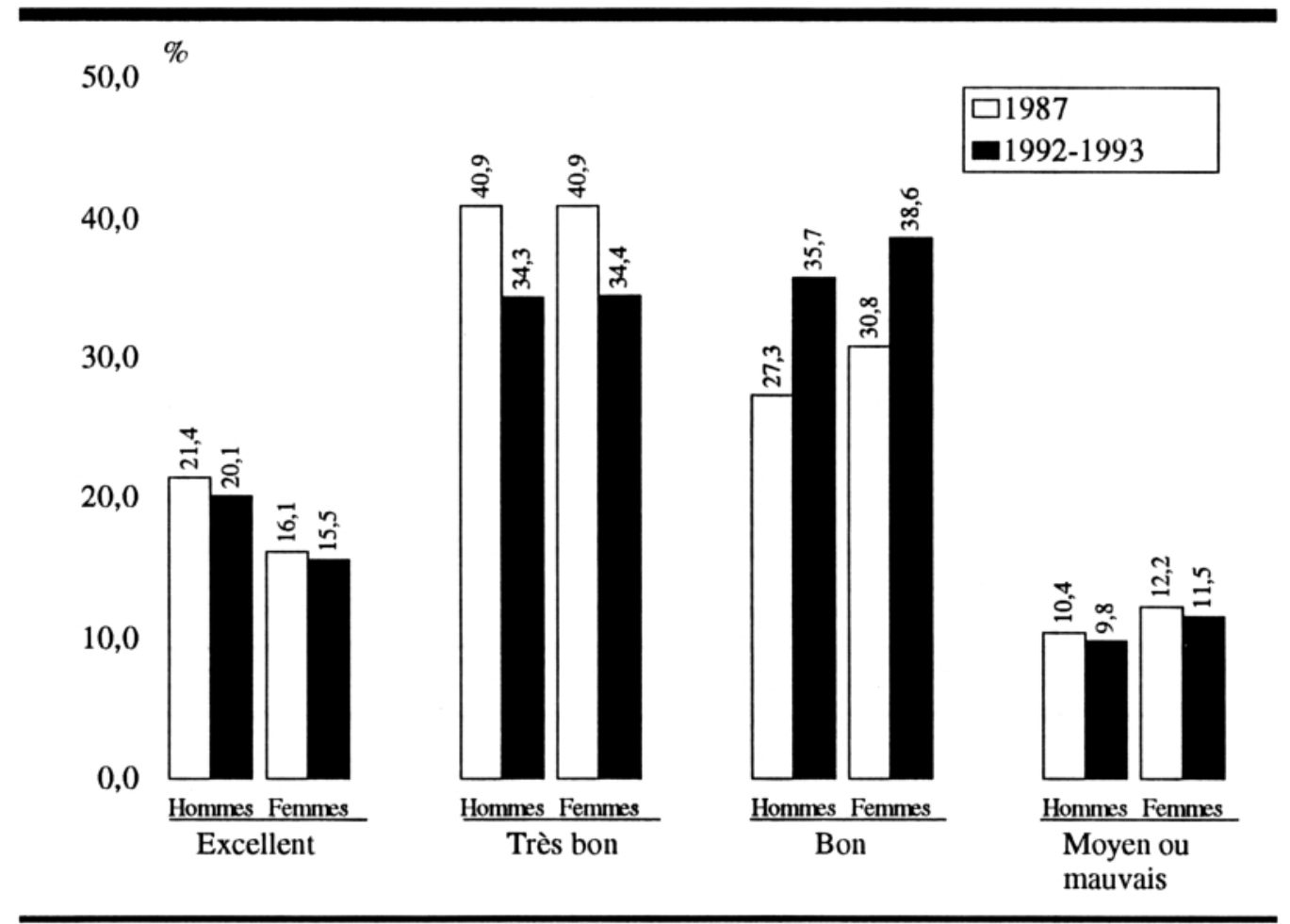




\subsubsection{Variations selon certaines caractéristiques socio-économiques}

La perception de l'état de santé varie en fonction de la scolarité relative. Selon l'enquête, les Québécois les moins scolarisés sont environ deux fois plus nombreux que les plus scolarisés à se considérer en moyenne ou en mauvaise santé (17\% c. 7\%) (tableau 11.2). Plus de trois personnes sur cinq (63\%) ayant une scolarité plus élevée évaluent leur état de santé comme étant excellent ou très bon comparativement à deux personnes de plus faible scolarité sur cinq (41\%). La même tendance s'observe chez les hommes et les femmes, mais les écarts semblent plus marqués chez ces dernières (tableau A. 11.2). 


\section{Tableau 11.2}

Perception de l'état de santé selon certaines caractéristiques socio-économiques, population de 15 ans et plus, Québec 1992-1993

$\underline{\text { Retour à la table des matières }}$

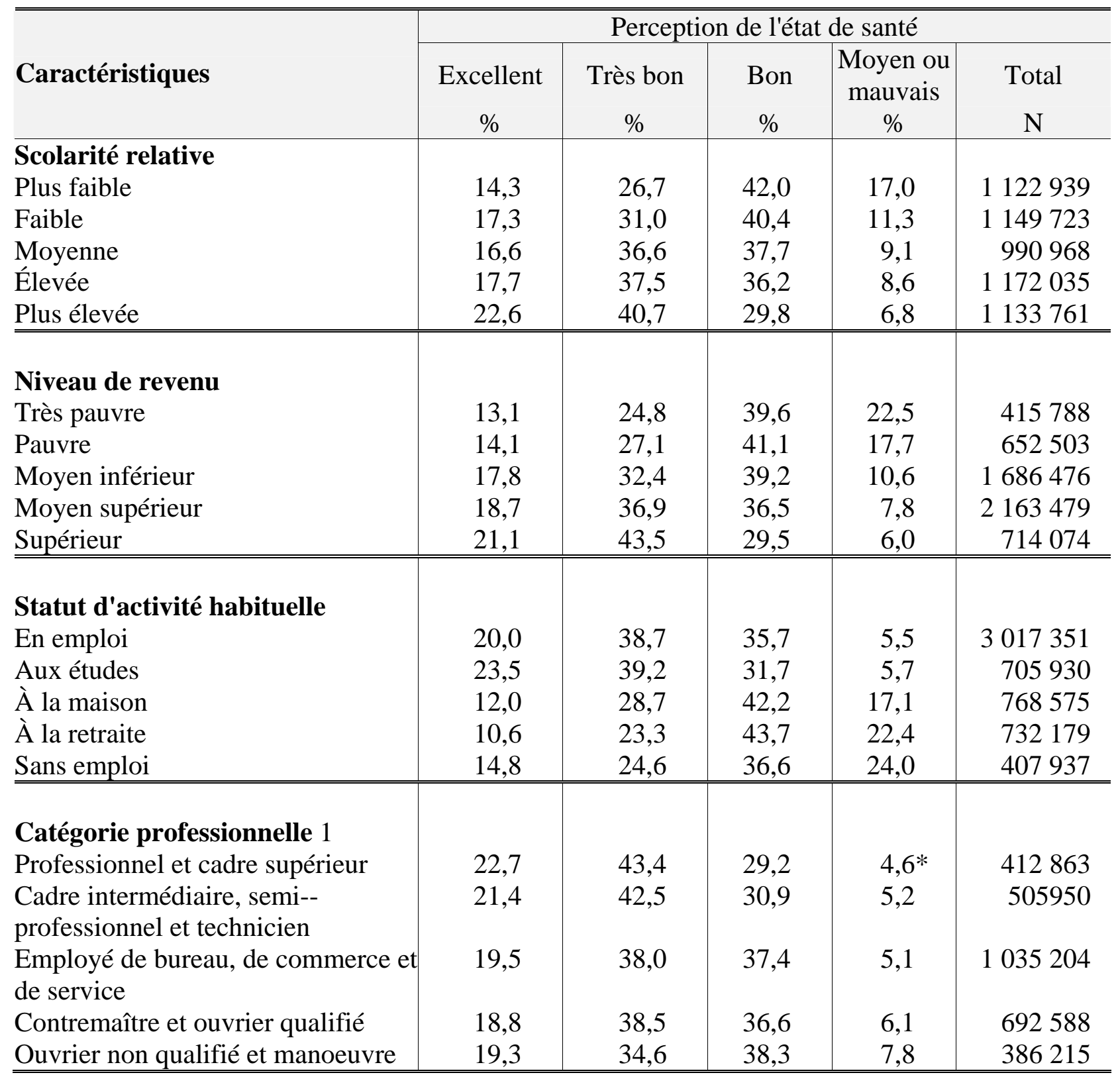

1 Population occupant un emploi au moment de l'enquête.

* Coefficient de variation entre $15 \%$ et $25 \%$; interpréter avec prudence. 
Plus le niveau du revenu est élevé, plus les gens déclarent avoir une excellente ou une très bonne santé. En revanche, les personnes qui font partie d'un ménage pauvre (18\%) ou très pauvre (23\%) sont beaucoup plus susceptibles de qualifier leur état de santé de moyen ou de mauvais que les personnes provenant de ménages dont le revenu se classe au niveau moyen supérieur (8\%) ou supérieur (6\%). Les différences sont semblables selon le sexe.

Le lien entre le statut d'activité habituelle et la perception de la santé pourrait être en partie un effet de l'âge. Les personnes qui évaluent le plus favorablement leur état de santé sont celles qui étudient, donc majoritairement des jeunes de 15-24 ans, et celles qui occupent un emploi, soit les personnes de 15 à 64 ans. Bien que les personnes sans emploi fassent partie du même groupe d'âge, le quart d'entre elles considèrent leur santé comme étant moyenne ou mauvaise ; cette catégorie d'activité comprend des personnes qui ne travaillent pas pour des raisons de santé. Une personne à la retraite sur cinq (22\%) s'estime en moyenne ou en mauvaise santé et $17 \%$ des personnes tenant maison, lesquelles sont en majorité des femmes âgées de 25 ans et plus (A. 11.3).

En ce qui concerne la catégorie professionnelle, elle ne semble pas jouer un rôle déterminant sur l'autoévaluation de la santé. Tout au plus, on note un écart significatif entre la perception de l'état de santé des professionnels et cadres supérieurs, et celle des ouvriers non qualifiés et manoeuvres. Les premiers sont plus nombreux que les seconds à qualifier leur santé de très bonne (43\% c. 35\%) et moins nombreux à la qualifier de bonne (29\% c. 38\%). Mais ces différences ne sont plus significatives lorsque les hommes et les femmes sont considérés séparément. Ces résultats ne s'appliquent qu'à la population qui occupe un emploi. 


\subsubsection{Variations selon certains autres déterminants}

\section{$\underline{\text { Retour à la table des matières }}$}

La perception de l'état de santé varie en fonction de comportements individuels tels que l'usage de la cigarette et la pratique d'activités physiques durant les loisirs. Les fumeurs réguliers se disent plus souvent en moyenne ou en mauvaise santé que les personnes qui n'ont jamais fumé (13\% c. 9\%), mais l'enquête ne montre pas de différence avec les anciens fumeurs ou les fumeurs occasionnels (tableau 11.3). Il y a aussi moins de fumeurs réguliers (45\%) que de personnes qui n'ont jamais fumé (57\%) et d'anciens fumeurs (54\%) qui s'estiment en excellente ou en très bonne santé. Les écarts apparaissent plus importants chez les hommes que chez les femmes (tableau A. 11.4).

C'est parmi les personnes dont la pratique de l'activité physique durant les loisirs est la plus fréquente (au moins trois fois par semaine) que se trouve la plus forte proportion d'individus (26\%) qui déclarent avoir une excellente santé. Les moins actifs, c'est-à-dire ceux qui pratiquent des activités physiques une fois par mois ou moins, sont environ deux fois plus nombreux (16\%) à qualifier leur santé de moyenne ou de mauvaise que les individus dont la pratique est plus fréquente (7\% à 8\%). Parmi ces derniers, les données ne montrent pas de différence significative entre ceux qui font des activités physiques de deux à trois fois par mois et ceux qui en font plus souvent. Les mêmes tendances s'observent chez les hommes et les femmes. 


\section{Tableau 11.3}

Perception de l'état de santé selon certains comportements individuels, population de 15 ans et plus, Québec 1992-1993

$\underline{\text { Retour à la table des matières }}$

\begin{tabular}{l|c|c|c|c|c}
\hline \multirow{2}{*}{$\begin{array}{l}\text { Comportements } \\
\text { individuels }\end{array}$} & \multicolumn{4}{|c}{ Perception de l'état de santé } \\
\cline { 2 - 6 } & Excellent & Très bon & Bon & $\begin{array}{c}\text { Moyen ou } \\
\text { mauvais }\end{array}$ & Total \\
& $\%$ & $\%$ & $\%$ & $\%$ & $\mathrm{~N}$ \\
\hline Type de fumeur & & & & & \\
Jamais fumé & 21,4 & 35,3 & 34,4 & 8,9 & 1730106 \\
Ancien fumeur & 17,3 & 36,5 & 35,9 & 10,3 & 1858191 \\
Fumeur occasionnel & 18,9 & 36,3 & 34,5 & 10,3 & 206356 \\
Fumeur régulier & 14,2 & 31,3 & 41,8 & 12,8 & 1659069 \\
\hline \hline $\begin{array}{l}\text { Activité physique de loisir } \\
\text { 3 fois/sem. et plus }\end{array}$ & 25,6 & 37,1 & 30,4 & 7,0 & 1396146 \\
2 fois/sem. & $.17,5$ & 40,6 & 34,8 & 7,1 & 720092 \\
1 fois/sem. & 17,8 & 36,5 & 37,6 & 8,2 & 815419 \\
2 2-3 fois/mois & 14,5 & 39,1 & 39,0 & 7,5 & 513361 \\
1 fois/mois et moins & 13,7 & 29,3 & 41,6 & 15,5 & 2072088 \\
\hline \hline
\end{tabular}

L'examen de l'indice de masse corporelle par rapport à la perception de l'état de santé permet d'observer une plus grande proportion d'hommes qui s'estiment en moyenne ou en mauvaise santé chez ceux qui ont un poids insuffisant (18\%) ou excédentaire (12\%) que chez ceux dont le poids est acceptable (8\%) (tableau 11.4). Il y a également plus de femmes à qualifier leur santé de moyenne ou de mauvaise parmi celles qui présentent un excès de poids que chez celles dont le poids est acceptable (18\% c. $10 \%$ ). Par contre, les femmes ayant un poids insuffisant ne sont pas plus nombreuses à évaluer leur santé comme étant moyenne ou mauvaise que les femmes de poids acceptable (11\% c. $10 \%)$. Les hommes et les femmes qui ont un poids accep- 
table déclarent plus souvent avoir une excellente santé que les hommes et les femmes dont le poids est excédentaire.

Les Québécois qui ont un niveau de soutien social élevé sont environ deux fois plus nombreux à se considérer en excellente santé que ceux dont le soutien social est faible (20\% c. 11\%) (tableau 11.5). En contrepartie, les personnes ayant un faible soutien social sont plus nombreuses (18\%) à se déclarer en moyenne ou en mauvaise santé que celles qui bénéficient d'un soutien social élevé (9\%). Les mêmes observations s'appliquent aux hommes et aux femmes (A. 11.4).

\section{Tableau 11.4}

Perception de l'état de santé selon l'indice de masse corporelle (IMC), hommes et femmes de 15 ans et plus, Québec 1992-1993

$\underline{\text { Retour à la table des matières }}$

\begin{tabular}{l|c|c|c|c|c}
\hline \multirow{2}{*}{$\begin{array}{l}\text { Indice de masse } \\
\text { corporelle }\end{array}$} & \multicolumn{5}{|c}{ Perception de l'état de santé } \\
\cline { 2 - 6 } & Excellent & $\begin{array}{c}\text { Très } \\
\text { bon }\end{array}$ & Bon & $\begin{array}{c}\text { Moyen ou } \\
\text { mauvais }\end{array}$ & Total \\
\hline Hommes & $\%$ & $\%$ & $\%$ & $\mathrm{~N}$ \\
Poids insuffisant & 17,3 & 28,2 & 37,0 & 17,5 & 156468 \\
Poids acceptable & 21,9 & 35,7 & 34,4 & 8,0 & 1782651 \\
Excès de poids & 17,2 & 32,1 & 38,5 & 12,1 & 777163 \\
\hline \hline Femmes & & & & & \\
Poids insuffisant & 15,8 & 37,4 & 35,7 & 11,1 & 442921 \\
Poids acceptable & 17,6 & 35,0 & 37,7 & 9,7 & 1719585 \\
Excès de poids & 9,4 & 30,5 & 42,5 & 17,6 & 569295 \\
\hline \hline
\end{tabular}




\section{Tableau 11.5}

Perception de l'état de santé selon l'indice de soutien social, population de 15 ans et plus, Québec 1992-1993

$\underline{\text { Retour à la table des matières }}$

\begin{tabular}{l|c|c|c|c|c}
\hline \hline \multirow{2}{*}{$\begin{array}{l}\text { Indice de masse } \\
\text { corporelle }\end{array}$} & \multicolumn{5}{|c}{ Perception de l'état de santé } \\
\cline { 2 - 6 } & Excellent & Très bon & Bon & $\begin{array}{c}\text { Moyen ou } \\
\text { mauvais }\end{array}$ & Total \\
& $\%$ & $\%$ & $\%$ & $\%$ & $\mathrm{~N}$ \\
\hline $\begin{array}{l}\text { Hommes } \\
\text { Élevé }\end{array}$ & 19,5 & 36,4 & 35,6 & 8,6 & 4363983 \\
Faible & 11,2 & 28,1 & 43,2 & 17,6 & 1102635 \\
\hline \hline
\end{tabular}

\subsection{Conclusions}

\subsubsection{Synthèse et pistes de recherche}

Par rapport à l'enquête Santé Québec 1987, la présente enquête révèle, dans l'ensemble, une stabilité dans la proportion de personnes qui qualifient leur santé d'excellente, de moyenne ou de mauvaise. Elle montre par ailleurs que les hommes et les femmes de 15 à 44 ans apprécient moins favorablement leur état de santé en 1992-1993 qu'en 1987. Cela s'exprime par une baisse de la proportion de personnes qui se considèrent en très bonne santé et une hausse de celles qui s'estiment en bonne santé. Ce déplacement vers un état de santé moins bon pourrait traduire une certaine morosité reliée au contexte socio-économique qui ne favorise pas toujours la population de ce groupe d'âge. Entre 45 et 64 ans, la tendance est plutôt vers une amélioration de la santé perçue, plus particulièrement chez les femmes. En 
ce qui concerne les personnes de 65 ans et plus, on peut parler d'une appréciation de leur état de santé qui est stable, sinon plus favorable.

Le rapport de l'enquête Promotion de la santé Canada 1990 fait état d'une stabilité pour les différents niveaux d'état de santé perçu entre 1985 et 1990 (Santé et Bien-être social Canada, 1993). Aucune différence significative n'est décelée non plus par groupe d'âge. Selon ce rapport, les enquêtes américaines (NHIS-HPDP) montrent que la proportion d'hommes et de femmes de 18 ans et plus ayant qualifié leur santé de mauvaise ou de moyenne n'a pas varié de façon significative entre 1985 et 1990. Marquée par la stabilité, l'évolution entre 1987 et 1992-1993 de la proportion de la population québécoise de 15 ans et plus se percevant en moyenne ou en mauvaise santé s'apparente ainsi à celle que l'on observe au Canada et aux États-Unis.

Les résultats de l'enquête montrent que des inégalités quant à l'état de santé perçu continuent d'exister en fonction des conditions de vie de la population. Ainsi, les personnes les plus susceptibles de qualifier leur santé de moyenne ou de mauvaise se retrouvent parmi celles qui sont les moins scolarisées, qui vivent dans les ménages pauvres ou très pauvres ou qui sont sans emploi. En raison de la relation entre ces différentes caractéristiques socio-économiques, ces personnes sont sans doute souvent les mêmes.

Par ailleurs, on observe un lien entre la perception de l'état de santé et l'usage de la cigarette d'une part, et, d'autre part, la pratique d'activités physiques durant les loisirs. Les personnes qui fument régulièrement sont plus nombreuses à se déclarer en moyenne ou en mauvaise santé comparativement à celles qui n'ont jamais fumé ; il en est de même pour les personnes les moins actives en regard de celles dont la fréquence des activités physiques est supérieure à une fois par mois.

L'indice de masse corporelle s'avère également être relié à la santé perçue. Les Québécois dont le poids est estimé insuffisant ou excé- 
dentaire qualifient plus souvent leur santé de moyenne ou de mauvaise que ceux dont le poids est acceptable.

Enfin, selon les données recueillies, il existe un lien entre le soutien social et l'autoévaluation de la santé. Les personnes ayant un soutien social faible évaluent plus négativement leur santé que celles dont le niveau de soutien est élevé.

Pour mieux comprendre l'évolution de la perception de l'état de santé, il y aurait lieu d'examiner l'évolution de ces déterminants. L'amélioration des conditions de vie et de certains comportements peut, par exemple, expliquer le fait que la perception de l'état de santé tend à s'améliorer à partir de 45 ans. Par ailleurs, le rapport entre la perception de la santé et la pratique d'activités physiques de loisir mérite d'être approfondi dans le but non seulement de déterminer les groupes cibles pour l'intervention, mais aussi la fréquence des activités physiques les plus favorables à la santé. Enfin, la relation entre certaines variables socio-économiques ou comportementales et cet indicateur de santé amène à soulever la question de l'ordre de causalité, à laquelle seule une étude longitudinale peut répondre.

\subsection{2 Éléments de réflexion pour la planification}

$\underline{\text { Retour à la table des matières }}$

Les disparités observées entre les groupes socio-économiques indiquent qu'il faut maintenir les objectifs de réduction des écarts dans le domaine de la santé, lesquels passent en grande partie par la réduction des inégalités sociales. Il importe également de poursuivre les efforts visant à réduire l'usage de la cigarette et à promouvoir l'activité physique, tout en gardant à l'esprit que les comportements individuels sont le plus souvent influencés par le milieu social, économique et culturel. 
En plus de permettre la définition de clientèles cibles pour la planification et la prise de décision, la perception de l'état de santé peut être mise à profit comme variable d'allocation des ressources (Pampalon et al. 1994 ; Piché, 1994). Actuellement, cet indicateur global de santé entre dans la composition d'un indice de besoin susceptible d'être utilisé pour l'allocation interrégionale des ressources dans le domaine de la santé physique (MSSS, 1994).

\subsection{Références bibliographiques}

$\underline{\text { Retour à la table des matières }}$

BARSKY, A. ; CLEARY, P.D. ; LERMAN, G.L. (1992). "Determinants of Perceived Health Status of Medical Outpatients", Social Science and Médecine, vol. 34, no 10, pp. 147-1154.

FYLKESNES, K. ; FORDE, O.H. (1992a). "The Tromso Study : Predictors of Self-Evaluated Health-Has Society Adopted The Expanded Health Concept ?", Social Science and Médecine, vol. 32, no 2, pp. 141-146.

FYLKESNES, K-; FORDE, O.H. (1992b). "Determinants and Dimensions Involved in Self-Evaluation of Health", Social Science and Médecine, vol. 35, no 3, pp. 271-279.

IDLER, E.L. ; ANGEL, R.J. (1990). "Self-Rated Health and Mortality in the NHANES-I Epidemiologic Followup Study", American Journal of Public Health, vol. 80, no 4, pp. 446-452. 
KUTNER, N.G. (1987). "Social Mes, Social Support, and Perceived Health Status Among Chronically Disabled People", Social Science and Medecine, vol. 25, no 1, pp. 29-34.

LIANG, J. ; BENNETT, J. ; WHITELAW, N. ; MAEDA, D. (1991). "The Structure of Self-Reported Physical Health Among the Aged in the United States and Japan", Medicare, vol. 29, no 12, pp. Il 61-1180.

LINN, B.S.; LINN, M. (1980). "Objective and Self-Assessed Health in the Old and very Old", Social Science and Medecine, vol. 14a, pp. 311-315.

MAYS, N. ; CHINN, S. ; HO, K.M. (1992). "Interregional variations in measures of health from the Health and Lifestyle Survey and their relation with indicators of health care need in England", Journal of Epidemiology and Community Health, vol. 46, pp. 38-47.

MINISTÈRE DE LA SANTÉ ET DES SERVICES SOCIAUX (1994). L'équité dans l'allocation interrégionale des ressources du champ de la santé physique, Rapport final du comité tripartite, ministère de la Santé et des Services sociaux, 96 pp.

PAMPALON, R. ; SAUCIER, A. ; BERTHIAUME, N. ; FERLAND, P. ; COUTURE, R. ; CARIS, P. ; FORTIN, L. ; LACROIX, D. ; KIROUAC, R. (1994). Des indicateurs de besoins pour l'allocation interrégionale des ressources, ministère de la Santé et des Services sociaux, document de travail, $72 \mathrm{pp}$.

PICHÉ, J. (1994). Indicateurs de besoins de services en santé physique : morbidité, mortalité, et conditions socio-économiques : revue de la littérature et analyse empirique, Service des indicateurs et systèmes socio-sanitaires, Direction des études et indicateurs, ministère de la Santé et des Services sociaux. 
SANTÉ ET BIEN-ÊTRE SOCIAL CANADA (1993). Enquête Promotion de la santé Canada 1990 : Rapport technique, ministère des Approvisionnements et Services Canada, Ottawa (Catalogue H39263/2-1990F).

SEGOVIA, J. ; BARTLETT, F.R. ; EDWARDS, A. (1989a). "'Me Association Between Self-assessed Health Status and Individual Health Practices", Canadian Journal of Public Health, vol. 80, pp. 3237.

SEGOVIA, J. ; BARTLETT, F.R. ; EDWARDS, A. (1989b). "An Empirical Analysis of the Dimensions of Health Status Measures", Social Science and Medecine, vol. 29, no 6, pp. 761-768.

WANNAMETHEE, G. ; SHAPER, A.G. (1991). "Self-Assessment of Health Status and Mortality in Middle-Aged British Men", International Journal of Epidemiology, vol. 20, no 1, pp. 239-245.

\section{Fin du texte}

